\title{
Multiple recurrent cerebral hydatidosis: A case report with review of literature
}

\author{
Nikhita $\mathrm{P}^{1}$, Vani $\mathrm{HN}^{2}$, Dattatreya $\mathrm{P}^{3}$, Nijaguna $\mathrm{N}^{4}$ \\ ${ }^{1}$ Post Graduate in Pediatrics, Indira Gandhi Institute of Child Health, Bangalore, ${ }^{2}$ Assistant Professor, Department of Paediatrics, Indira Gandhi \\ Institute of Child Health, Bangalore, ${ }^{3}$ Post Graduate in Pediatrics, Indira Gandhi Institute of Child Health, Bangalore, ${ }^{4}$ Profesor of Paediatrics, \\ Indira Gandhi Institute of Child Health, Bangalore, India
}

\section{A B S T R A C T}

Echinococcisis is an emerging zoonotic parasitic disease worldwide and is a significant health burden among developing nations. Cystic hydatidosis is more commonly found in lungs and liver but rarely cerebral involvement can occur. Multiple hydatid cysts of brain are uncommon and may be either primary or secondary. Hydatid cyst shoud be considered as a differential diagnosis for cystic lesions of the brain. Here we are reporting a 8 year old boy with multiple cerebral hydatid cysts recurring within three months after surgical rupture of a primary large cerebral hydatid cyst.

Key words: Cerebral Echinococcosis, Hydatidosis

\section{INTRODUCTION}

Hydatid cyst is caused by the infestation of the larvae of Echinococcus granulosis. The definite hosts are various carnivores, the most common being dog. The intermittent hosts are various mammals. Liver is the most common organ, followed by lungs. In children, hydatid cyst is common in lungs, because of the possible bypass of lymphatics, hydatid cyst have been found in brain, heart, and kidneys also. Multiple cerebral hydatid cysts in brain are uncommon. It might be primary or secondary. Cerebral hydatid cyst is a rare manifestation of Echinococcosis accounting for $2-3 \%$ of intracranial masses and 50 to $75 \%$ of cases are seen in children and young adults. ${ }^{1}$ Only a few of them are associated with extracranial manifestations of the disease making the exact pathogenesis difficult to explain leaving a few debatable schools of thought. ${ }^{2-4}$ There are case reports of successful therapy with albendazole. Surgery remains the treatment of choice despite the newer modalities. $^{5}$

\section{CASE REPORT}

A-8 yr old boy presented to us with complaints of fever, altered sensorium and progressive loss of speech over the last one month. The child on examination had abnormal movements involving the left upper limb. On examination his vitals were stable, Glasgow coma scale score of 9/15. Hypotonia in all limbs with exaggerated reflexes. Signs of meningeal irritation were present. Other vital systems examinations were within normal limits. Neuroimaging was suggestive of hydrocephalus with multiple intracranial multiloculated hydatid cysts with significant peri-lesional edema (Figure 1).

This boy had presented to another hospital one year back with complaints of progressively worsening of right hand weakness and headache over past three months. At that time his vitals were stable and sensorium normal, but there was definite weakness in the right upper limb with no associated organomegaly according to the medical documents they possessed. MRI done had revealed a well defined intraventricular cystic lesion involving the left lateral ventricle (Figure 2), with possible differentials of arachnoid cyst or neuro-epithelial cyst. There was mass effect in the form of effacement of adjacent cerebral sulci, left lateral ventricle, midline shift and descending transtentorial herniation. Ultrasound abdomen and chest $\mathrm{X}$ ray were normal. Cysto-peritoneal shunt was performed, later after histo-pathology report suggestive of hydatid cyst, cyst 
excision was done after two days. There was no evidence of anaphylaxis or allergic reactions at the time of surgery. Child improved neurologically after surgery.

Three months later, child presented again with acute onset fever and altered sensorium. Re-evaluation revealed hydrocephalus with multiple multi-loculated cysts. Child underwent extra-ventricular drain in the frontal region. The drain was in-situ for 8 days during which time the sensorium of the child improved. Child was discharged on anti-epileptics and was advised to continue albendazole.

Child was symptomatically better for two months and later presented to us with the above complaints. Detailed history and evaluation revealed that this was a case of multiple recurrent cerebral hydatid cyst which had occurred due to surgical rupture of intracranial solitary hydatid cyst, which was mistaken as arachnoid cyst. Medical treatment with albendazole had not helped this child and child succumbed in our hospital after three days.

\section{DISCUSSION}

Hydatidosis is caused by Echinococcus granulosis. It occurs commonly in dogs, primary host. Intermediate hosts like man get infected accidentally by ingestion of faeces. The eggs hatch in the intestine and burrow through to the portal circulation. The embryos that survive are able to survive in many tissues most commonly liver and lungs. Even after the pulmonary filter, a few still make it to the systemic circulation and reach any tissue such as brain, heart and bones etc.

Cerebral hydatidosis is a rare manifestation of the disease. Ninety percent tend to be isolated, ${ }^{6}$ supratentorial and intraparenchymal and in the middle cerebral artery territory, ${ }^{7}$ however a few of them could be multiple and tend to involve the ventricles. The association of cerebral cysts with extracranial involvement is debatable. Some authors reported a high concomitant cysts while others didn't. In clinical practice, not many extracranial cysts are reported and the plausible explanation is brain being a soft organ, cysts tend to grow faster and postoperative or eradicative therapy takes care of the clinically or radiologically insignificant smaller cysts at other extracranial sites. $^{8}$

Brain hydatid cysts could be primary or secondary. Secondary brain hydatid cysts tend to be multiple and are usually dislodged from a heart focus or rupture of a primary brain focus.' They are rare and constitute only $2-4 \%$ of total cases. Cerebral hydatid cysts are more common among children and young adults. Extracranial hydatid cysts are to be looked for through chest X ray and abdominal USG.

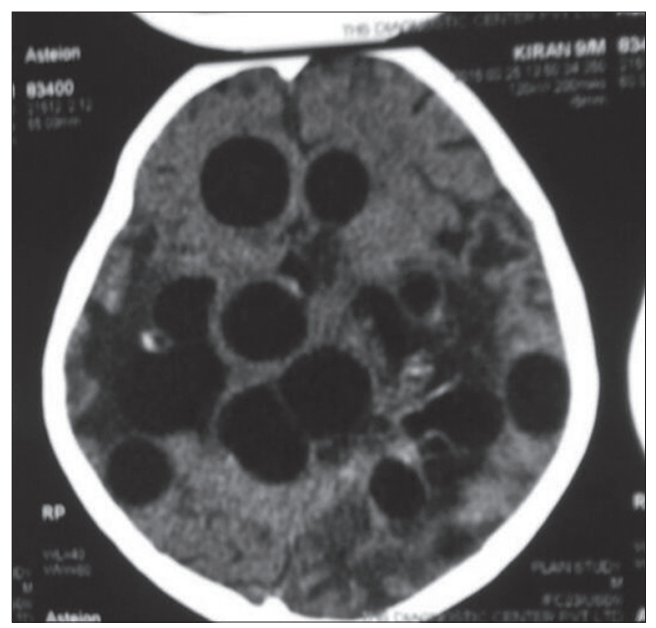

Figure 1: Hydrocephalus with multiple intracranial multiloculated hydatid cysts with significant peri-lesional edema

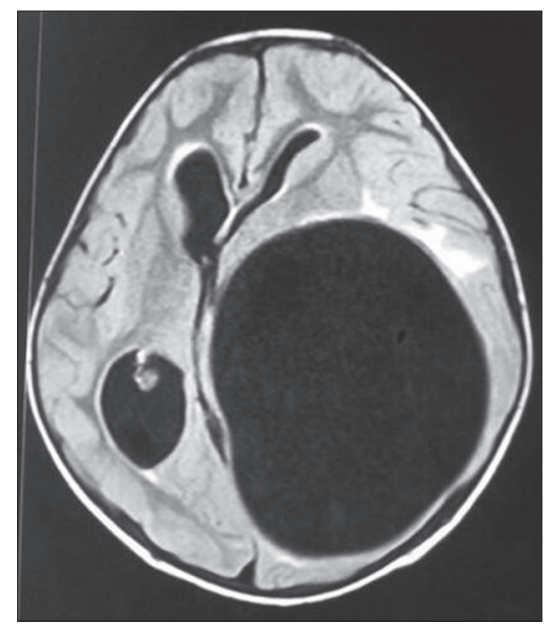

Figure 2: Well defined intraventricular cystic lesion involving the left lateral ventricle. There is mass effect in the form of effacement of adjacent cerebral sulci, left lateral ventricle, midline shift and descending

Heart ultrasound is specifically indicated in children, where the occurrence of primary cerebral hydatid cysts may imply a shunt between the right and left side of the heart as incidences are high where there is associated PDA.

Posterior fossa locations are rare. The wall of the cyst consists of inner endocysts, which produces the scolises, outer ectocyst and the host response, fibrous wall - pericyst.

\section{PRESENTATION, DIAGNOSIS AND MANAGEMENT}

Most cerebral hydatid cysts are acquired during the childhood and are presenting insidiously in childhood and young adult life. ${ }^{10}$ The usual presenting symptoms in early adulthood are signs of raised intracranial pressure like headache, vomiting, diplopia, ataxia, abducens nerve palsy, seizures, altered sensorium, speech defects, hemiparesis 
and coma in severe case. ${ }^{10}$ Multiple intracranial hydatid cysts are often considered malignant disease as case series suggesting fatal outcome in such cases post operatively. ${ }^{11}$

Diagnosis is confirmed by histopatholgical examination of the cyst, though serological tests like Casoni and Weinberg test, ELISA, indirect haemaglutination may be additional but not useful in intracranial hydatid cyst as brain tissue evokes minimal response. MRI considered superior to CT brain in diagnosing the lesion. A solitary cyst appears well defined, spherical smooth, and thin walled with inner density similar to CSF. Surrounding parenchymal edema and ring enhancement suggestive of brain abscess and other cystic neoplasms. ${ }^{11}$ MRI helps in exact cyst location and surrounding cyst edema, characteristics of cyst, and helps in surgical intervention. ${ }^{12}$

Treatment of this condition involves both surgical and medical modalities. Medical therapy is favored in small or inoperable cases. Albendazole, either alone or in combination is used. A total duration of four weeks or sometimes prolonged therapy is advised, with authors favoring prolonged therapy. It is reported that albendazole causes disappearance of lesions in about $48 \%$ cases and reduction in the size in about $28 \%$ which is well supported by Magnetic resonance studies. ${ }^{13,14}$

Surgery remains the mainstay of treatment in intracranial hydatid cyst. Dowling's procedure being most commonly used involves continuous saline irrigation in cyst brain interface causing complete delivery of the cyst without rupture. Rupture spontaneously or during surgery is known to cause fatal anaphylactic shock and recurrence. ${ }^{15}$

Recurrence is a major concern and is reported to be as high as $25 \%$ in a case series. ${ }^{15}$ Recurrences are managed with anti-helimenthic therapy and surgery. In our present case report, in view of progressive neurological deficit and the size of the cyst, neurosurgical intervention is considered with adjunct albendazole therapy. Early and complete evaluation of intracranial cystic lesion evaluation is important to prevent seeding elsewhere and adjunct prolonged medical therapy is essential in intracranial hydatidosis.

\section{REFERENCES}

1. Kovoor JME, Thomas R D, Chandrashekhar HS, Jayakumar PN, Pillai S, Shankar SK. Neurohydatisis. Australas Radiol 2007;51:406-11.

2. Cavusoglu H, Tuncer C, Ozdilomac A, Aydin Y. Multiple Intracranial Hydatid cysts in boy. Turk Neurosurg 2009;19(2):203.

3. Ray M, Singhi PD, Pathak A and Khandelwal NK. Primary multiple intracerebral echinococcosis in a young child. J Trop Pediatr. 2001;51(1): 59-61.

4. Erashin Y, Mutluer S, Guzelbag E. Intracranial hydatid cysts in children. Neurosurg. 1993;33(2):219-224.

5. Reddy DR. Managing cerebral and cranial hydatid disease. Neurol India 2009;57:116-8.

6. Andronikou S, Welman $\mathrm{C}$ and Kader E. Classic and unusual appearance of hydatid disease in children. Ped Radiolog 2002; 32(11): 817-828.

7. Mancuso P, Noubari BA and Gurunsinghe NT. Multiple primary cerebral hydatid cysts. Br J Neurosurg 1997;11(3): 248-249.

8. Zahawi HM, Hameed OK and Abalkhail AA. The possible role of the age of the human host in determining the localization of hydatid cysts. Annals Trop Med Parasitol 1999;93(6): 621-627.

9. Khaldi M, Mohamed S, Kall J and Khouja N. Brain hydatidosis: Report on 117 cases. Child's Nerv Syst 2000;16: 765-769.

10. Popli MB and Khudale B. Primary multiple hydatid cysts of the brain. Australasian Radiol 1998; 42(1):90-91.

11. Nemati A, Kamagarpour, Rashid M, Nazari S.S. Giant Cerebral Hydatid cyst in a child. A case report with review of literature. BJMP 2010;3(3):a338.

12. Shamam $O E$, Amer $T$, Atta M A. Magnetic resonance imaging of simple and infected hydatid cysts of the brain. Magn Reson Imaging 2001;19: 965-974.

13. Horton RJ. Albendazole in treatment of human cystic echinococcosis: 12 years of experience. Acta Trop 1997; 64:79.

14. Neumayr A, Troia G, de Bernardis C, Tamarozzi F, Goblirsch S, Piccoli L, et al. Justified concern or exaggerated fear: The risk of anaphylaxis in percutaneous treatment of cystic echinococcosisa systematic literature review. PLoS Negl Trop Dis 2011; 5:e1154.

15. Junghanss T, Menezes da Silva A, Horton J, Chiodini PL and Brunetti E. Clinical management of cystic echinococcosis: State of the art, problems and perspectives. Am J Trop Med Hyg 2008; 79:301-311.

\footnotetext{
Authors Contribution:-

NP - Prepared first draft of manuscript; VHN - Concept and design of case report, reviewed case report; DP - Concept literature search; NN - Concept and reviewed case report.

Source of Support: Nil, Conflict of Interest: None declared.
} 